九州大学学術情報リポジトリ

Kyushu University Institutional Repository

\title{
Amino Acid Composition of Grape Cultivars ( Vitis Spp.) in Japan
}

Shiraishi, Shin-ichi

University Farm, Faculty of Agriculture, Kyushu University

https://doi.org/10.5109/23976

出版情報 : 九州大学大学院農学研究院紀要. 36 (1/2)，pp.117-122，1991-10. Kyushu University バージョン：

権利関係 : 


\title{
Amino Acid Composition of Grape Cultivars ( Vitis Spp.) in Japan
}

\author{
Shin-ichi Shiraishi \\ University Farm, Faculty of Agriculture, \\ Kyushu University, Kasuyamachi, Fukuoka 811-23
}

(Received July 29, 1991)

\begin{abstract}
The concentration of free amino acids and other chemical constituents in 75 grape cultivars at maturity were examined. The amino acid concentrations were found to be in decreasing order of arginine, proline, threonine and alanine in Vitis vinifera cultivars and alanine, arginine, threonine and proline in Vitis labrusca cultivars.

The concentration of total amino acid was 5,083 $\mu \mathrm{mol}$ in 'Campbell Early' and 3,391 $\mu \mathrm{mol}$ in 'Yates', both Vitis labrusca and table grapes. The amounts of total amino acids in vinifera and wine grape cultivars were low while those of table grape cultivars were high in organoleptic sweet amino acids such as threonine, serine, proline, glycine, alanine, etc.
\end{abstract}

\section{INTRODUCTION}

The amino acids in table grapes are important as a source of the good taste contributors. The importance of amino acids in contributing to the taste of foodstuffs was first recognized by Ikeda who discovered that monodium L-glutamate was the essential component of the taste-imparting ingredients of traditional Japanese food seasoners such as sea tangle. The taste of traditional Japanese foods such as soy sauce was presumed to be due to the amino acids which were released from the natural proteins during the course of fermentation (Kirimura et al. 1969).

Amino acids are used in food processing not only to enhance the nutritive value, but also to enhance or improve the taste of natural characterestics of foodstuffs. The taste of amino acids and their relative stuffs. The taste of amino acids and their relative contribution to the taste of foods have been the topic of extensive investigations ; studies have been undertaken on the distribution, isolation, and elucidation of the chemical structures of amino acids and some of their properties in foods.

The amino acid contents in Vitis vinifera (Castor et al. 1953, 1956 ; Kliewer 1968, 1970 ; Ough 1968, 1969) and V.labrusca (Gallander 1974 ; Kliewer 1969 ; Kluba et al.) grapes were reported.

This paper presents the data on the composition of the free amino acids in many vinifera and labrusca cultivars cultivated in Japan.

\section{MATERIALS AND METHODS}

About $50 \mathrm{~g}$ of ripe berries were sampled from ten clusters in the center portion of the randomly selected vines in the experimental vineyards of Fukuoka Horticulture Experiment Station, Fukuoka, and the Akitsu Branch of the Fruit Tree Research Station Akitsu in 1977 and 1978. Afrer removing the seeds, $50 \mathrm{~g}$ samples were ground with quarz sand in a triturator, $200 \mathrm{ml}$ of deionized water was added to the macerate 
and then clarified by centrifugation at $10.000 \mathrm{G}$ and $10 \mathrm{~min}$. The supernatant was decanted, and the residue was reextracted several times with deionized water and filtered through Toyo No. 5B filter paper. Preparation and determination for amino acid content were examined by the ion exchange column chromatography, and the analyses of sugars and organic acids were done through the procedure described by Shiraishi et al. $(1980 ; 1986)$.

\section{RESULTS AND DISCUSSION}

Table 1 shows the concentrations of the free amino acid compositions in the ripe grapes. The total sugars, total and free acids of the grape cultivars examined are presented in Table 2 .

The most abundant free amino acids in all 75 grape cultivars at maturity were alanine, arginine, and proline, constituting 20.9, 27.7 and $15.3 \%$ of the total free amino acids, respectively. Amounts ranged from 9 to $2.482 \mu \mathrm{mol}$ per $100 \mathrm{~g}$ fresh berries for alanine, 34 to $1,045 \mu \mathrm{mol}$ for arginine, and 14 to $1,037 \mu \mathrm{mol}$ for proline. Threonine, serine, and glutmic acid values ranged between 1 and $110 \mu$ mol, and their amount varied with cultivars.

The most abundant free amino acids in Vitis vinifera cultivars were arginine, proline, threonine and alanine with the average percentages of 35.4, 21.2, 11.7 and 9.7, respectively (Table 3). The average amounts of these amino acids were 295.2, 176.0, 97.1 and $81.2 \mu \mathrm{mol}$ per $100 \mathrm{~g}$ fresh berries, respectively.

Likewise, the most abundant free amino acids in labrusca cultivars were alanine arginine, threonine and proline with the average percentages of 31.7, 18.2, 17.7 and 9.0, and amounts of 549.5, 269.8, 261.8 and $132.9 \mu \mathrm{mol}$ per $100 \mathrm{~g}$ fresh berries, respectively.

Labrusca grape 'Campbell Early' had the highest content of total amino acids $(5,083 \mu \mathrm{mol}$ per $100 \mathrm{~g}$ fresh berries), followed by same labrusca 'Yates' $(3,391 \mu \mathrm{mol})$. Average total free amino acid levels in labrusca and vinifera grapes were 1,479 and 833 $\mu$ mol, respectively.

The grape cultivars were arbitrarily grouped into low-, medium-and high-amino acid groups with total amino acids of 2,139 and $1,000 \mu \mathrm{mol}$ as thresholds. The highamino acid group included labrusca cultivars and high quality table grapes. The medium-amino acid group included many hybrid cultivars and table grapes. The lowamino acid group included vinifera cultivars and many wine grapes.

Amino acids were also divided into 4 groups by their chemical constructions : The first group was the monoamino monocarboxylic acid which included glycine, alanine, serine, cystine, threonine, methionine, valine, leucine isoleucine, phenylalanine and tyrosine. The second group was the polyamino monocarboxylic acid which included arginine, histidine and lysine. The third group was the monoamino dicarboxylic acid which included aspartic acid and glutamic acid. The fourth group was the iminocarboxylic acid which included proline and hydroxyproline. Hydroxyproline was rarely present in the grape cultivars examined in this study.

The amino acid compositions divided into 4 groups with the 75 cultivars are shown in Table 2. Higher concentration of monoamino monocarboxylic acids seemed to be responsible for the high content of total amino acids in some grape cultivars. Polyamino monocarboxylic among which arginine is the major component, are basic 
Table 1. Comparison of amino acid composition in the 75 cultivars* of grapes $\left(^{* 2}\right.$ : vinifera, $3:$ labrusca)

\begin{tabular}{|c|c|c|c|c|c|c|c|c|c|c|c|c|c|c|c|c|c|c|}
\hline \multirow{3}{*}{ Cultivar } & & & & & & & & & & & & & & & & & & \\
\hline & & & & & & & & & & & & & & & & & & \\
\hline & $\mathrm{Asp}$ & $\mathrm{Thr}$ & Ser & $\mathrm{C}$ Glu & Pro & Gln & $\mathrm{Ala}$ & Cys & Val & Met & Ile & Leu & Tyr & Phe & Lys & His & Arg & Total \\
\hline Campbell early ${ }^{3}$ & 52 & 583 & 85 & 125 & 238 & 80 & 2.482 & 6 & 82 & 12 & 45 & 75 & 12 & 35 & 38 & 88 & 1.045 & 5,083 \\
\hline Yates3 & 31 & 906 & 120 & 136 & 128 & 25 & 1,499 & 0 & 46 & 2 & 20 & 35 & 3 & 20 & 9 & 19 & 392 & 3,391 \\
\hline Pusa seedless' & 90 & 640 & 110 & 215 & 440 & 9 & 411 & 9 & 50 & 6 & 21 & 69 & 0 & 34 & 10 & 45 & 560 & 2,719 \\
\hline Neo Muscat' & 32 & 593 & 71 & 78 & 476 & 19 & 240 & 0 & 62 & 16 & 39 & 46 & 8 & 31 & 73 & 75 & 735 & 2,591 \\
\hline Bath" & 60 & 740 & 90 & $0 \quad 89$ & 35 & 11 & 923 & 1 & 28 & 7 & 10 & 12 & 5 & 5 & 8 & 24 & 408 & 2.456 \\
\hline Pione $^{2}$ & 26 & 696 & 106 & 91 & 70 & 20 & 530 & 2 & 56 & 11 & 25 & 40 & 6 & 36 & $\mathrm{Y}$ & 45 & 518 & 2,287 \\
\hline Cannon Hall Muscat $^{2}$ & 15 & 190 & 106 & 68 & 620 & 20 & 175 & 1 & 60 & 6 & 39 & 73 & 4 & 25 & 27 & 54 & 736 & 2,221 \\
\hline Kokuhō $\bar{o}^{3}$ & 40 & 313 & 156 & 63 & 618 & 28 & 634 & 0 & 44 & 6 & 26 & 28 & 4 & 19 & 4 & 14 & 191 & 2,188 \\
\hline Kyohō & 20 & 188 & 106 & 64 & 134 & 24 & 548 & 2 & 110 & 15 & 60 & 79 & 20 & 40 & 30 & 44 & 655 & 2,139 \\
\hline Buffalo" & 24 & 243 & 74 & 84 & 427 & 19 & 617 & 0 & $4 \%$ & 2 & 12 & 33 & 2 & 16 & 11 & 20 & 275 & 1,901 \\
\hline Himrod seedless' & 30 & 480 & 98 & 78 & 178 & 15 & 445 & 0 & 46 & 6 & 27 & 33 & 6 & 20 & 10 & 33 & 330 & 1.835 \\
\hline Russki Concord ${ }^{2}$ & 56 & 204 & 71 & 68 & 1,037 & 10 & 122 & 1 & 35 & 6 & 23 & 29 & 1 & 1 & 7 & 20 & 88 & 1,779 \\
\hline Kendaia $^{3}$ & 13 & 197 & 16 & 40 & 58 & 24 & 1,115 & 0 & 35 & 1 & 15 & 25 & 10 & 10 & 8 & 10 & 60 & 1,637 \\
\hline Nehelescol ${ }^{2}$ & 18 & 124 & 45 & 45 & 844 & 6 & 98 & 0 & 15 & 10 & 12 & 15 & 0 & 15 & 5 & 17 & 337 & 1,606 \\
\hline Red Millennium' & 16 & 165 & 66 & 70 & 350 & 17 & 500 & 0 & 35 & 1 & 11 & 22 & 1 & 19 & 15 & 25 & 293 & 1,606 \\
\hline Romulus ${ }^{2}$ & 30 & 181 & 83 & 125 & 175 & 10 & 105 & 0 & 31 & 0 & 14 & 30 & 0 & 10 & 6 & 22 & $4 \times 2$ & 1,604 \\
\hline Schyler ${ }^{y}$ & 22 & 177 & 51 & 57 & 392 & 15 & 318 & 0 & 29 & 1 & 8 & 24 & 0 & 8 & 8 & 17 & 393 & 1.518 \\
\hline Tachikawa No. 3' & 25 & 280 & 35 & 63 & 70 & $\mathrm{Y}$ & 425 & 0 & 15 & 0 & 6 & 13 & 0 & 10 & 6 & 19 & 470 & 1,446 \\
\hline Violet Uehara ${ }^{2}$ & 41 & 332 & 91 & 42 & 26 & 9 & 541 & 0 & 20 & 1 & 8 & 17 & 0 & 9 & 5 & 14 & 264 & 1,420 \\
\hline Italia $^{2}$ & 30 & 256 & 35 & 60 & 94 & 12 & 107 & 1 & 32 & 8 & 20 & 32 & 9 & 20 & 39 & 36 & 615 & 1,406 \\
\hline Interlaken seedless' & 15 & 120 & 44 & 104 & 733 & 9 & 115 & 0 & 65 & 0 & 10 & 16 & 0 & 6 & 8 & 15 & 145 & 1,405 \\
\hline Delaware" & 11 & 201 & 47 & 72 & 86 & 21 & 467 & 0 & 23 & 1 & 8 & 21 & 8 & 14 & 11 & 12 & 392 & 1,395 \\
\hline $\mathrm{Ko} \bar{o} \bar{u}^{2}$ & 15 & 83 & 62 & 27 & 531 & 15 & 138 & 1 & 15 & 1 & 9 & 19 & 1 & 1 & 9 & 16 & 424 & 1,367 \\
\hline Black Olympia' & 30 & 247 & 78 & 61 & 100 & 10 & 455 & 0 & 16 & 0 & 9 & 15 & 4 & 10 & 10 & 20 & 295 & 1,360 \\
\hline Coma Neagra ${ }^{2}$ & 13 & 177 & 52 & 51 & 251 & 12 & 230 & 0 & 30 & 4 & 10 & 19 & 3 & 8 & 7 & 20 & 465 & 1,352 \\
\hline Alden $^{9}$ & 24 & 355 & 41 & 70 & 66 & 10 & 388 & 0 & 21 & 1 & 7 & 18 & 3 & 10 & 6 & 19 & 285 & 1,324 \\
\hline Cabernet flanc ${ }^{2}$ & 8 & i 4 & 45 & 35 & 594 & 9 & 174 & 0 & 20 & 0 & 8 & 15 & 0 & 8 & 6 & 17 & 300 & 1,313 \\
\hline Tachikawa No. 5" & 31 & 275 & 90 & 60 & 55 & 15 & 373 & 6 & 35 & 0 & 20 & 25 & 8 & 24 & 10 & 23 & 220 & 1,270 \\
\hline Pierce' & 27 & 268 & 62 & 45 & 23 & 15 & 54.5 & 0 & 31 & 1 & 11 & 23 & 8 & 19 & 8 & 20 & 139 & 1,245 \\
\hline Urbana' & 11 & 225 & 62 & 67 & 66 & 8 & 398 & 0 & 26 & 2 & 5 & 15 & 5 & 23 & 5 & 17 & 241 & 1,176 \\
\hline Mase No. $5^{3}$ & 10 & 182 & 34 & 34 & 156 & 31 & 265 & 0 & 6 & 0 & 5 & 10 & 0 & 6 & 12 & 15 & 405 & 1,171 \\
\hline Keuka $a^{3}$ & & 70 & 48 & 10 & 15 & 0 & 300 & 0 & 40 & 2 & 14 & 31 & 11 & 16 & 15 & 19 & 435 & 1,051 \\
\hline Hiro Hamburg' & 10 & 30 & 70 & 98 & 58 & 10 & 115 & 0 & 38 & 6 & 21 & 46 & 2 & 1 & 16 & 36 & 490 & 1,047 \\
\hline Anab-e shahi ${ }^{2}$ & 25 & 250 & 59 & 44 & 105 & 10 & 140 & 0 & 28 & 5 & 15 & 15 & 2 & 15 & 7 & 20 & 281 & 1,021 \\
\hline Concord' & 28 & 223 & 52 & 42 & 37 & 7 & 410 & 0 & 18 & 3 & 8 & 9 & 1 & 12 & 2 & 11 & 150 & 1,013 \\
\hline Captivator-' & 18 & 122 & 23 & 19 & 195 & 24 & 167 & 0 & 45 & 5 & 14 & 35 & 17 & 66 & 12 & 32 & 206 & 1,000 \\
\hline Ishihara wase $^{3}$ & 18 & 174 & 20 & 28 & 34 & 10 & 523 & 0 & 19 & 6 & 6 & 11 & 6 & 10 & 5 & 9 & 110 & 989 \\
\hline Sheridan $^{3}$ & 32 & 149 & 39 & 59 & 40 & 7 & 394 & 0 & 24 & 4 & 8 & 15 & 6 & 8 & 6 & 11 & 121 & $96 \mathrm{X}$ \\
\hline Nyora $^{2}$ & 15 & 140 & 35 & 30 & 255 & 9 & 130 & 0 & 25 & 2 & 10 & 10 & 0 & 6 & 9 & 8 & 245 & 929 \\
\hline Köshū sanjaku ${ }^{2}$ & 14 & 159 & 42 & 44 & 305 & 6 & 82 & 0 & 10 & 1 & 6 & 11 & 0 & 9 & 7 & 15 & 204 & 915 \\
\hline Olympia' & $3 \mathrm{Y}$ & 137 & 96 & 49 & 130 & 10 & 260 & 0 & 29 & 3 & 13 & 25 & 3 & 16 & 2 & 7 & 96 & 913 \\
\hline Katta Kurgan ${ }^{2}$ & 15 & 148 & 20 & 34 & 58 & 6 & 46 & 0 & 11 & 2 & 6 & 16 & 2 & 20 & 11 & 20 & 475 & 890 \\
\hline Purple Damascus ${ }^{2}$ & 20 & 104 & 29 & 30 & 315 & 9 & 110 & 0 & 25 & 2 & 8 & 13 & 2 & 6 & 8 & 13 & 173 & 867 \\
\hline Hanover $^{2}$ & 20 & 156 & 45 & 28 & 60 & 11 & 370 & 0 & 1.5 & 2 & 7 & 16 & 6 & 20 & 7 & 12 & 78 & 853 \\
\hline Black queen ${ }^{2}$ & 40 & 101 & 41 & 29 & 151 & 5 & 182 & 0 & 15 & 2 & 11 & 25 & 1 & 6 & 4 & 7 & 231 & 851 \\
\hline Rose queen' & 19 & 122 & 50 & 37 & 55 & $\mathrm{a}$ & 195 & $\mathrm{n}$ & 20 & 2 & 8 & 16 & 5 & 10 & 6 & 15 & 265 & 838 \\
\hline Pecocede Marengre ${ }^{2}$ & 31 & 66 & 41 & 22 & 23 & 7 & 56 & 0 & 13 & 0 & 8 & 20 & 0 & 0 & 6 & 9 &, 514 & $\mathrm{Xl} 8$ \\
\hline Naples $^{3}$ & 14 & 95 & 45 & 16 & 35 & 9 & 360 & 2 & 12 & 2 & 10 & 20 & 2 & 14 & 2 & 11 & 117 & 766 \\
\hline Muscat Hamburg ${ }^{2}$ & 15 & 78 & 30 & 36 & 68 & I & 95 & 0 & 16 & 1 & 12 & 18 & 6 & 8 & 9 & 16 & 355 & 764 \\
\hline Black prince' & 27 & 100 & 30 & 30 & 39 & 6 & 132 & 0 & 7 & 2 & 9 & 8 & 0 & 6 & 10 & 12 & 328 & 746 \\
\hline Roode IIanepoot ${ }^{2}$ & 17 & 112 & 71 & 47 & 58 & 5 & 134 & 0 & 21 & 2 & 10 & 20 & 2 & 7 & 6 & 13 & 188 & 713 \\
\hline Gros Colman' & 29 & 36 & 21 & 22 & 109 & 3 & 39 & 0 & 19 & 1 & 13 & 25 & 3 & 13 & 8 & 16 & 348 & 706 \\
\hline l'inot blanc ${ }^{2}$ & 2 & 56 & 9 & 5 & 195 & 2 & 16 & 0 & 16 & 0 & 6 & 11 & 2 & 6 & 11 & 11 & 333 & 681 \\
\hline Rasaki² & 38 & 100 & 51 & 28 & 117 & 6 & 81 & 0 & 32 & 2 & 10 & 13 & 2 & 11 & 10 & 11 & 132 & 644 \\
\hline Shasslas rose' & 25 & 54 & 30 & 18 & 102 & 3 & 73 & 0 & 18 & 0 & 9 & 15 & 0 & 5 & 5 & 13 & 269 & 639 \\
\hline Ontario & 10 & 136 & 22 & 25 & 21 & 6 & 212 & 0 & Y & 0 & 2 & 9 & 2 & 3 & 2 & 10 & 158 & 627 \\
\hline Niagara3 & 10 & 141 & 24 & 15 & 24 & 10 & 220 & 0 & 12 & () & 7 & 11 & 10 & 22 & 5 & 10 & 100 & 621 \\
\hline Monukka ${ }^{2}$ & 23 & 71 & 23 & 35 & 215 & 6 & 70 & 0 & 11 & 2 & 8 & 16 & 0 & 9 & 6 & 14 & 111 & 620 \\
\hline Merlot ${ }^{2}$ & 8 & 40 & 28 & 45 & 338 & 8 & $\mathrm{i} \mathrm{i}$ & 0 & 16 & II & 6 & 9 & 0 & 0 & 5 & 5 & 34 & 619 \\
\hline Golden Muscat' & 7 & 128 & 21 & 31 & 30 & 10 & 145 & 0 & 11 & 0 & 8 & 17 & 0 & 11 & 10 & 15 & 160 & 604 \\
\hline Canada Muscat' & 11 & 101 & 43 & 21 & 44 & $\mathrm{Y}$ & 119 & 1 & 10 & 2 & 6 & 13 & 4 & 15 & 5 & 12 & 148 & 574 \\
\hline Rizamat $^{2}$ & 25 & 57 & 18 & 5 & 50 & 3 & 54 & 0 & 11 & 0 & 3 & 8 & 0 & 5 & 6 & 12 & 289 & 548 \\
\hline Athens' & 16 & 62 & 16 & 41 & 41 & 11 & 145 & 2 & 12 & 0 & 6 & 11 & 9 & 14 & 6 & 10 & 115 & 517 \\
\hline Gros Sémillon" & 5 & 44 & 29 & 16 & 119 & 6 & 65 & 0 & 13 & 0 & 6 & 9 & 0 & 6 & 6 & 8 & 142 & 474 \\
\hline Riesling Italico ${ }^{2}$ & 9 & 42 & 37 & 7 & 44 & 2 & 69 & 0 & 6 & 4 & 9 & 17 & 0 & 3 & 6 & 13 & 205 & 473 \\
\hline Black II Iamburg ${ }^{2}$ & 6 & 24 & 13 & 6 & 24 & 6 & 20 & 2 & 13 & 2 & 11 & 20 & I & $\mathrm{Y}$ & 7 & $\mathrm{Y}$ & 282 & 457 \\
\hline Flame Tokay ${ }^{2}$ & 9 & 56 & 15 & 16 & 134 & 6 & 43 & 0 & Y & 0 & 3 & 6 & 0 & 5 & 4 & 8 & 124 & 438 \\
\hline Pizzutello Bianco & 15 & 41 & 19 & 16 & 37 & 1 & 36 & 0 & 14 & 1 & 8 & 15 & 0 & 15 & 7 & 9 & 193 & 427 \\
\hline Black Cornichohon ${ }^{2}$ & $\mathrm{Y}$ & 31 & 14 & 14 & 81 & 6 & 27 & 0 & $i$ & 0 & 5 & 9 & 0 & 4 & 6 & 5 & 181 & 399 \\
\hline Ryugan $^{2}$ & 10 & 55 & 30 & 16 & 95 & 15 & 62 & 0 & 7 & 0 & 5 & 8 & 1 & 6 & I & 8 & 78 & 397 \\
\hline Königin drr Weingarten ${ }^{2}$ & 14 & 55 & 20 & 25 & 42 & 6 & 45 & 0 & 11 & 0 & $\mathrm{Y}$ & 8 & 0 & 10 & 6 & 6 & 112 & 369 \\
\hline Centennial $^{2}$ & 20 & 65 & 58 & 15 & 25 & 5 & 45 & 0 & 6 & 0 & 4 & 4 & 1 & 5 & 4 & 8 & 75 & 340 \\
\hline Chardonnay $^{2}$ & 10 & 28 & 14 & 6 & 65 & 5 & 9 & 0 & 10 & 0 & 6 & 10 & 0) & 4 & 6 & 5 & 150 & 328 \\
\hline Grüner Sylvaner ${ }^{2}$ & 17 & 24 & 25 & 10 & 23 & 8 & 23 & 0 & 17 & 0 & 28 & 42 & 0 & 15 & 3 & 5 & 40 & 280 \\
\hline Olivette rose $\mathrm{e}^{2}$ & 15 & 30 & 12 & 11 & 14 & 5 & 21 & 0 & 6 & 1 & 5 & 5 & 0 & 3 & 6 & 8 & 135 & 277 \\
\hline
\end{tabular}


Table 2. Sugar and acid contents, and constructive distributions and organoleptic characteristics of amino acids in the 75 cultivars ${ }^{* *}$ of grapes $\left({ }^{*} \mu \mathrm{mol} / 100 \mathrm{~g}\right.$ of fresh weight $\left(^{* * 2}\right.$ : vinifera, ${ }^{3}$ :labrusca)

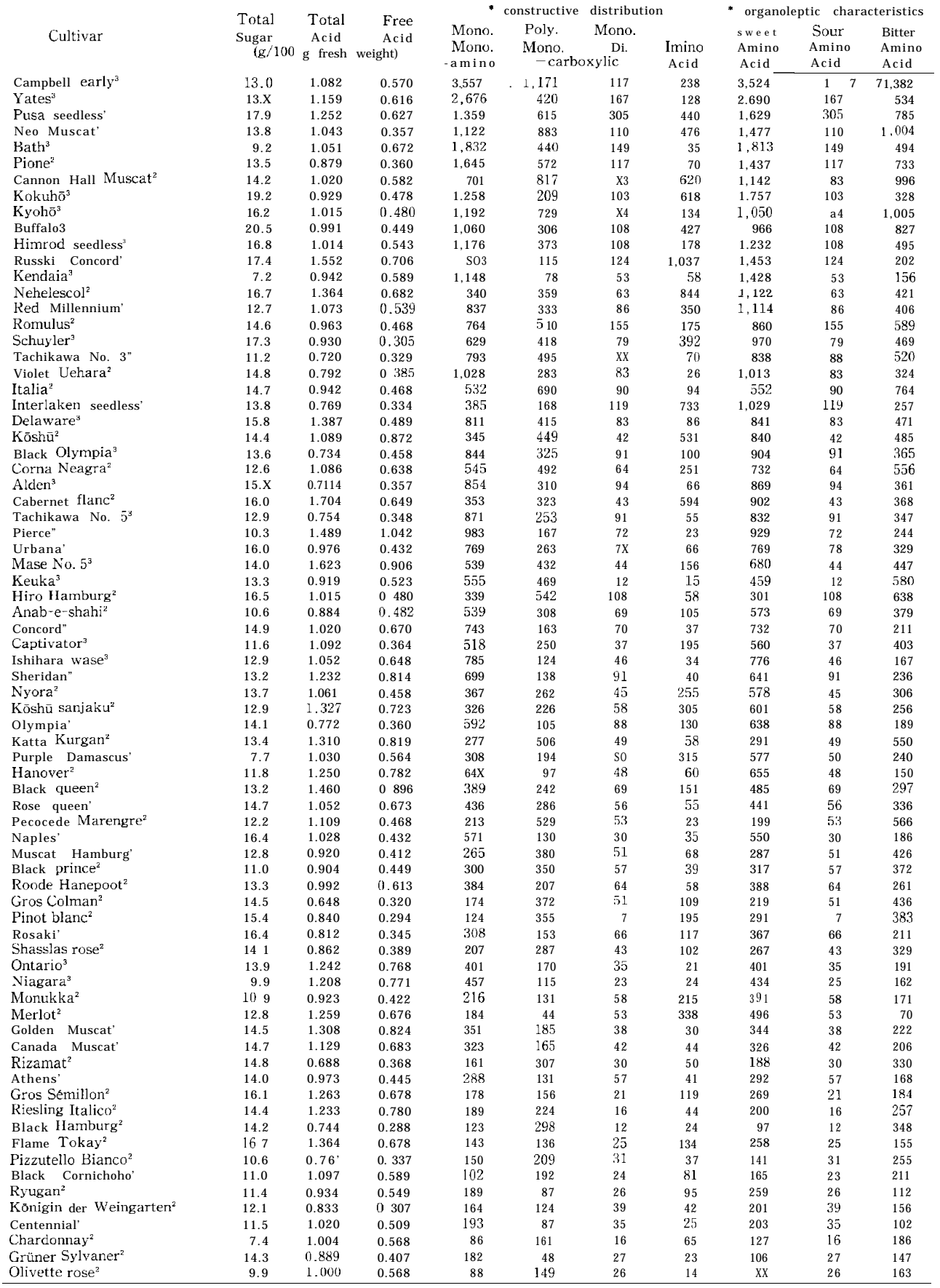


Table 3. Amounts of the major free amino acids and percentages in Vitis vinifera. and labrusca. cultivar graoups

\begin{tabular}{lcccc}
\hline Group & Alanine & Arginine & Proline & Threonine \\
vinifera & $81.2^{*}$ & 295.2 & 176.0 & 97.1 \\
& $9.7^{* *}$ & 35.4 & 21.2 & 11.7 \\
labrusca & 549.5 & 269.8 & 132.9 & 261.8 \\
& 31.7 & 18.2 & 9.0 & 17.7 \\
\hline
\end{tabular}

* Amount : $\mu \mathrm{mol}$ per $100 \mathrm{~g}$ fresh berries

* * Percentage

amino acids, and are contained in many vinifera cultivars. 'Pusa seedless' had the highest content of monoamino dicarboxylic acids among 75 grape cultivars examined. Among grape cultivars with relatively high amounts of monoamino dicarboxylic acids were 'Yates'(167), 'Romulus'(155), 'Bath'(149), 'Russki concord'(124), 'Interlaken seedless'(119), 'Campbell Early' and 'Pione'(117), 'Neo Muscat'(110), 'Buffalo', 'Himrod seedless', and 'Hiro Hamburg'(108), and 'Kokuhō'(103 $\mu \mathrm{mol})$. Iminocarboxylic acid group contained only proline. There seemed no relationship among species in the concentration of proline. The proline contents were 1,037, 844, 733, 620 and $518 \mu \mathrm{mol}$ per $100 \mathrm{~g}$ fresh berries in 'Russki Concord, 'Nehelescol', 'Interlaken seedless', 'Cannon Hall Muscat' and 'Kokuho' grapes, respectively.

Amino acids are classified into three types-sweet, sour, and bitter amino acids by their organoleptic characterestics. Table shows the concentration of them in 75 cultivars. Threonine, serine, proline, glycine, alanine, cystine, tyrosine and lysine are important sweet taste contributors. These amino acids were contained in many labrusca cultivars and table grapes in high quantity. Aspartic-and glutamic acid contribute to sour taste. Cultivars containing relatively abundant concentrations of sour amino acids are grapes with good taste. Valine, methionine, isoleucine, leucine phenylalanine, histidine and arginine have a bitter taste accompanied by slight sweetness. These amino acids were found to be contained more in the vinifera than in the labrusca cultivars, and the average percentages were 22.4 in the labrusca and 45.5 in the vinifera cultivars.

Amounts of total sugar contents ranged from 7.2 to $20.5 \mathrm{~g}$ per $100 \mathrm{~g}$ fresh berries. Higher total sugar contents of 20.5, 19.2, 17.9, 17.4 and $17.3 \mathrm{~g}$ per $100 \mathrm{~g}$ fresh berries were observed on 'Buffalo', 'Kokuho', 'Pusa seedless', 'Russki Concord' and 'Schuyler', respectively. 'Kendaia', 'Chardonnay', and 'Purple Damascus' contained only small amounts of total sugars with respective 7.2, 7.4, and 7.7 g. There seemed no relationship among species in the concentration of total sugar, showing the average content of $13.8 \mathrm{~g}$ for cultivars belonging to both species. Most abundant total acid contents of 1 . 704, 1.552, 1.489, 1.387 and $1.364 \mathrm{~g}$ per $100 \mathrm{~g}$ fresh berries were found in 'Cabernet franc', 'Russki Concord', 'Pierce', 'Delawere' and 'Nehelescol', respectively. Amounts of free acids ranged from 0.288 to $1.042 \mathrm{~g}$ fresh berries. There seemed no relationship among species in the concentration of free acid. The results presented here are in accordance with Kluba et al. (1978). and Shiraishi et al. (1980).

It was concluded that the grape berries have many different patterns in free amino acids, and the table grape cultivars have the abundant content of sweet amino acids according to organoleptic characteristics. These results will further provide some 
meaningful suggestions for the improvement of the quality of grape products containing useful amino acids.

\section{REFERENSES}

Castor, J. G. B. 1953 The free amino acids of must and wines. I. Microbiological estimation of fourteen amino acids in California grape musts. Food Res. 18 : 139-145

Castor, J. G. B. and T. E. Archer 1956 Amino acids in must and wines, proline, serine and threonine. Amer. J.Enol. $7: 19-25$

Callander, F. 1974 Chemistry of grapes and other fruits as the raw materials involved in winemaking. P. 11-49. In : A. D Webb (ed.) Chemistry of winemaking. Adv. in chem. series. Vol. 137

Kirimura, J., A. Shimizu, A. Kirizuka, T. Ninomiya and N. Katsuya 1969 The concentration of peptides and amino acids to the taste of foodstuffs. J. Agr. Food Chem. 17: 689-695

Kliewer, W. M. 1968 Changes in the concentration of free amino acids in grape berries during maturation. Amer. J. Enol.Vitic. 19 : 166-174

Kliewer, W. M. 1969 The free amino acids and other nitrogenous substances of table varieties. J.Food Sci. $34: 274-278$

Kliewer, W. M. 1970 Free amino acids and other nitrogenous fractions in wine grapes. J.Food Sci. 35: 17-21

Kluba, R. M., L. R. Mattick and L. R. Hackler 1978 Changes in the free and total amino acid composition of several Vitis labruscana grape varieties during maturation. Amer. J.Enol. Vitic. $29: 102^{-}$ 111

Ough, C. S. 1968 Proline content of grapes and wines. Vitis. 7:321-331

Ough, C. S. and 0. Buston 1969 A review of amino acid analytical methods and their application to grapes and wine. Wines and vines 50: 50-58

Shiraishi, S. 1980 Fundamental study for grape breeding in the environmental controlled system. I. Accumulation of organic acids in berries of main grape cultivars during their maturation. Bull. Fukuoka Hort. Exp. Sta. 18 : 9917

Shiraishi, S., T. Sumi and K. Notsuka 1986 Changes in the chemical constituents of three table grape varieties (Vitis vinifera L. $\times$ V.labrusca L.) during maturation in Japan. J. Japan. Soc. Hort. Sci. 55: $15-21$ 